\section{Validação de indicadores do consumo de alimentos e bebidas obtidos por inquérito telefônico em Belém, Pará, Brasil}

\author{
Validation of food and beverage indicators \\ obtained by telephone survey in Belém, \\ Pará State, Brazil
}

Alice Cristina Medeiros das Neves 1 Lidyane Andrea Amaral Gonzaga 1 Irland Barroncas Gonzaga Martens 1 Erly Catarina Moura ${ }^{2}$
1 Universidade Federal do Pará, Belém, Brasil. 2 Ministério da Saúde, Brasília, Brasil.

Correspondência A. C. M. Neves

Universidade Federal do Pará. Trav. Djalma Dutra 146, Belém, PA 66113-010, Brasil. lica_medeiros@hotmail.com

\begin{abstract}
The aim of this study was to evaluate the relative validity of indicators of food and beverage intake obtained from the telephone interview surveillance system (VIGITEL). A random sample ( $n=$ 100) was evaluated from the total sample of approximately two thousand adults studied by the system in 2009 in Belém, Pará State, Brazil. The indicators were protective factors (adequate consumption of fruit, vegetables, and leafy vegetables) and riskfactors (consumption of saturated fat, soft drinks, and alcoholic beverages) for chronic noncommunicable diseases. The telephone interview results were compared with those of three 24-hour recalls (reference standard). The reference standard showed underestimation in the indicators' frequency, except for soft drinks and alcoholic beverages. The mean consumption frequencies were generally higher in the exposed group interviewed by the VIGITEL system. We cannot conclude that the VIGITEL system is not a good indicator of consumption, since the reference standard also shows limitations. Nevertheless, its use as a surveillance tool in Brazil is justifiable.
\end{abstract}

Food Consumption; Diet Surveys; Epidemiologic Surveillance

\section{Introdução}

A alimentação é um fator importante na promoção e manutenção da saúde, durante a vida. Seu papel como determinante das doenças crônicas não transmissíveis (DCNT) já ocupa uma posição importante em atividades de prevenção da saúde 1 . A epidemia crescente das doenças não transmissíveis se associa com o consumo insuficiente de frutas, legumes e verduras, com o aumento no consumo de alimentos fonte de gorduras saturadas e de açúcares refinados, além do consumo abusivo de bebidas alcoólicas 2 .

Estimativas da Organização Mundial da Saúde (OMS) apontam que, em 2001, as DCNT foram responsáveis por cerca de $60 \%$ do total de mortes ocorridas no mundo e por aproximadamente $46 \%$ da carga global de doenças ${ }^{1}$. No Brasil, as estimativas são semelhantes. Dados de 2006 apontam essas enfermidades como a principal causa de óbito na população brasileira, destacando-se as doenças do aparelho circulatório $(29,4 \%)$ e as neoplasias $(15,1 \%) 3$.

Com o objetivo de monitorar a frequência e a distribuição dos principais determinantes das DCNT no Brasil, o Ministério da Saúde começou a operar, em 2006, o Sistema de Vigilância de Fatores de Risco e Proteção para Doenças Crônicas por Inquérito Telefônico (VIGITEL). Esse sistema se baseia em inquéritos telefônicos, utilizando questionário eletrônico ${ }^{4}$, realizados em amostras probabilísticas da população adulta com 
telefone fixo, residente em cada uma das 26 capitais de estados brasileiros e no Distrito Federal.

O VIGITEL, apesar de ter sido validado em São Paulo por Monteiro et al. 5, necessita de validação nas diferentes regiões do país, em virtude da diversidade regional e cultural, característica peculiar de um país tão imenso e heterogêneo como o Brasil. Tais diversidades devem ser observadas para o aprimoramento do VIGITEL como instrumento de avaliação de consumo de alimentos e bebidas, para que os erros sejam minimizados e os dados obtidos estejam o mais próximo possível da ingestão real ${ }^{6,7}$. Nesse sentido, em 2009, foi realizado estudo de validação dos indicadores alimentares monitorados pelo VIGITEL em Belém, Estado do Pará, maior capital da Região Norte.

\section{Métodos}

Trata-se de estudo transversal de validade relativa, realizado numa subamostra composta por cem pessoas, número recomendado para estudos deste tipo ${ }^{8}$, sendo 36 homens e 64 mulheres. Esses indivíduos compuseram a amostra de pouco mais de duas mil pessoas maiores de 18 anos, residentes na cidade de Belém, entrevistadas pelo VIGITEL em 2009.

OVIGITEL considera um mínimo de duas mil entrevistas por cidade com intervalo de $95 \%$ de confiança e erro máximo de $2 \%$ na obtenção de qualquer estimativa. A amostra é feita em duas etapas: sorteio de linha telefônica residencial listada no cadastro telefônico e sorteio de um indivíduo adulto de cada casa, o qual tenha concordado em participar do estudo 9 . No período de 13 de fevereiro a 30 de abril de 2009, indivíduos entrevistados pelo VIGITEL compuseram uma amostra total de três réplicas, resultando em 304 entrevistas completas. Num prazo mínimo de 15 e máximo de 30 dias após a entrevista do VIGITEL, novas tentativas de contato com essas pessoas foram feitas para o estudo de validade. Dentre os que foram encontrados, que concordaram em participar deste estudo e completaram as três entrevistas telefônicas necessárias para a validação, conseguiu-se uma subamostra de 100 indivíduos, o que, para o total da população estudada, atendia à recomendação de Willett \& Lenart 8 para estudos desta natureza, ainda que limitado para os sexos separadamente.

$\mathrm{O}$ inquérito telefônico aplicado pelo VIGITEL 10 constou de entrevista em que se utilizou questionário eletrônico; com questões sobre características demográficas e socioeconômicas, características do padrão de alimentação, entre outras.
O estudo de validade relativa consistiu na realização, por entrevista telefônica, de três recordatórios alimentares de 24 horas, nos quais cada indivíduo foi questionado sobre informações referentes ao tipo e quantidade de todos os alimentos e bebidas consumidos nas últimas 24 horas.

As questões do VIGITEL foram: "Em quantos dias da semana o(a) Sr(a) costuma comer feijão?"; "Em quantos dias da semana o (a) Sr(a) costuma comer carne vermelha (boi, porco, cabrito)?"; "Quando o(a) Sr(a) come carne vermelha com gordura, costuma tirar sempre o excesso de gordura visivel ou comer com a gordura? Ou não come carne vermelha com muita gordura?"; "Em quantos dias da semana o (a) Sr(a) costuma comer frango/galinha?"; "Quando o(a) Sr(a) come frango/galinha com pele, costuma tirar sempre a pele, comer com a pele ou não come pedaços de frango com pele?", "Em quantos dias da semana $o$ (a) Sr(a) costuma tomar leite? (não vale 'leite' de soja)"; "Quando o (a) Sr(a) toma leite, que tipo de leite costuma tomar?"; "Em quantos dias da semana o(a) Sr(a) costuma tomar refrigerante (ou suco artificial)?"; "Que tipo: normal, diet/light/ zero ou ambos?"; "Num dia comum, quantas copos o(a) Sr(a) toma de suco de frutas natural?"; "Em quantos dias da semana o(a) Sr(a) costuma comer frutas?"; "Num dia comum, quantas vezes o(a) Sr(a) come frutas?"; "Em quantos dias na semana, o(a) Sr(a) costuma comer pelo menos um tipo de verdura ou legume (alface, tomate, couve, cenoura, chuchu, berinjela, abobrinha)? (não vale batata, mandioca ou inhame)"; "Em quantos dias da semana, o(a) Sr(a) costuma comer salada de alface e tomate ou salada de qualquer outra verdura ou legume cru?"; "Num dia comum, o(a) Sr(a) come esse tipo de salada no almoço, no jantar ou no almoço e no jantar?"; "Em quantos dias da semana, o(a) Sr(a) costuma comer verdura ou legume cozido junto com a comida ou na sopa, como, por exemplo, couve, cenoura, chuchu, berinjela, abobrinha, sem contar batata, mandioca ou inhame?"; "Num dia comum, o(a) Sr(a) come verdura ou legume cozido no almoço, no jantar ou no almoço e no jantar?”; "O(a) Sr(a) costuma consumir bebida alcoólica?"; "Com que frequência o(a) Sr(a) costuma ingerir alguma bebida alcoólica?", "Nos últimos 30 dias, o Sr chegou a consumir cinco ou mais doses de bebida alcoólica em uma única ocasião?" (para homens) ou "Nos últimos 30 dias, a Sra chegou a consumir quatro ou mais doses de bebida alcoólica em uma única ocasião?" (para mulheres).

Os recordatórios foram aplicados no decorrer de duas semanas, sendo dois deles referentes a dias de semana e um referente a domingo ou feriado. Ao final do relato de cada indivíduo 
os itens de interesse (frutas, legumes e verduras; carne vermelha, frango, leite, refrigerantes e bebidas alcoólicas) não citados foram questionados diretamente quanto ao consumo no dia avaliado. Em relação a frutas, legumes e verduras, em situações particulares, perguntava-se sobre quantidade consumida. Com referência à carne vermelha, perguntava-se sobre a existência ou não de gordura visível e se esta era ou não ingerida; em situações particulares, perguntava-se sobre a quantidade consumida. O mesmo acontecia com o frango em relação ao consumo da pele. No caso do leite, perguntava-se se o tipo era integral ou desnatado. Para o consumo de refrigerante, perguntava-se sobre o tipo (com ou sem restrição de açúcar). E, para bebida alcoólica, perguntavase sobre a quantidade consumida.

Com base nos três recordatórios de 24 horas (R24h), calculou-se a frequência semanal do consumo de alimentos e de bebidas de cada indivíduo, multiplicando-se por 2,5 o número referente aos recordatórios de dia de semana e por 2,0 o número referente aos recordatórios realizados no domingo ou feriado, para cada alimento e bebida.

O estudo de validade relativa consistiu em comparar os resultados obtidos no VIGITEL com os resultados dos três R24h (padrão de referência - PR), considerando a proporção de indivíduos expostos a determinado evento. Os eventos avaliados foram os indicadores do comportamento alimentar associados à proteção ou risco para doenças crônicas não transmissíveis. O indicador considerado de proteção foi o consumo regular de frutas, legumes e verduras ( $\geq 5$ dias por semana); os indicadores de risco foram consumo habitual de gordura saturada ( $\geq 2$ dias por semana, de leite com teor integral de gordura e carne vermelha com gordura visível ou carne de frango com pele); consumo regular de refrigerante com açúcar (pelo menos uma lata ou copo $\geq 5$ dias por semana), consumo abusivo de bebidas alcoólicas (cinco ou mais doses para o homem e quatro ou mais doses para a mulher, em uma mesma ocasião nos últimos 30 dias) ( $\geq 1$ dia por semana), segundo Monteiro et al. 5 .

O grau de concordância individual entre as duas entrevistas (VIGITEL e PR) foi avaliado pelo cálculo do coeficiente kappa: acima de 0,80 indica concordância quase perfeita; entre 0,61 e 0,80 indica concordância substancial; entre 0,41 e 0,60 concordância moderada; entre 0,21 e 0,40 concordância razoável; abaixo de 0,21 significa concordância fraca 11,12.

Para avaliar a concordância do VIGITEL em classificar corretamente os indivíduos expostos ao evento segundo o padrão de referência, calculou-se o percentual de indivíduos que receberam o diagnóstico de exposição (sensibilidade) e não exposição (especificidade) na entrevista telefônica. Foram calculados os falsos positivos e falsos negativos, que indicam, respectivamente, a porcentagem de casos não expostos na entrevista da validação, mas que se classificaram como expostos no VIGITEL, e a porcentagem de casos expostos na entrevista da validação, mas que se classificaram como não expostos no VIGITEL. Os cálculos da sensibilidade, da especificidade, do falso positivo e do falso negativo são recomendados em estudos de validade relativa, em face das características dos indicadores avaliados 13,14.

Adicionalmente, calculou-se a mediana da frequência semanal do consumo de alimentos e de bebidas, estimada a partir dos três R24h entre os indivíduos classificados pelo VIGITEL como expostos ou não à frequência crítica de consumo, definida pelos indicadores de comportamento alimentar associados à proteção ou risco para DCNT (cinco ou mais dias da semana para frutas, legumes e verduras, e de refrigerante com açúcar; pelo menos dois dias por semana de excesso de gordura - carne ou frango - e de leite integral; e pelo menos um dia por semana de consumo de bebidas alcoólicas). O significado estatístico das diferenças entre os dois grupos foi avaliado empregando-se o teste das diferenças entre medianas $(\mathrm{p}<0,05)$.

Para a análise dos dados foi utilizado o aplicativo SPSS, versão 13.0 (SPSS Inc., Chicago, Estados Unidos).

Por se tratar de entrevista por telefone, o consentimento livre e esclarecido foi substituído pelo consentimento verbal obtido por ocasião dos contatos telefônicos com os entrevistados. $\mathrm{O}$ estudo de validação foi aprovado pelo Comitê de Ética em Pesquisa em Seres Humanos do Núcleo de Medicina Tropical da Universidade Federal do Pará, e o projeto VIGITEL foi aprovado pelo Comitê Nacional de Ética em Pesquisa para Seres Humanos do Ministério da Saúde.

\section{Resultados}

A população avaliada apresentava idade igual a $43,7$ anos (desvio padrão $=14,1), 10,7(4,5)$ anos de estudo, índice de massa corporal referido igual a 25,2 $(4,6) \mathrm{kg} / \mathrm{m}^{2}$ e realizava $4,0(0,9)$ refeições por dia. Não houve diferença entre homens e mulheres (Tabela 1), e os valores foram semelhantes aos encontrados na população estudada pelo VIGITEL, respectivamente 43,0 $(16,4)$ anos de idade, 10,9 $(4,6)$ anos de estudo e $25,2(4,6)$ $\mathrm{kg} / \mathrm{m}^{2}$ (dados não mostrados).

A Tabela 2 compara a frequência de indicadores de consumo alimentar estimado com base 
Tabela 1

Idade, escolaridade, índice de massa corporal referido (IMC) e número de refeições diárias - média (desvio padrão) da população estudada.

\begin{tabular}{lccc}
\hline & $\begin{array}{c}\text { Total }(\mathbf{n}=100) \\
\text { Média (desvio-padrão) }\end{array}$ & $\begin{array}{c}\text { Homens }(\mathbf{n}=\mathbf{3 6}) \\
\text { Média (desvio-padrão) }\end{array}$ & $\begin{array}{c}\text { Mulheres ( } \mathbf{n}=64) \\
\text { Média (desvio-padrão) }\end{array}$ \\
\hline Idade (anos) & $43,7(14,1)$ & $43,9(12,9)$ & $43,6(14,8)$ \\
Escolaridade (anos) & $10,7(4,5)$ & $10,3(5,0)$ & $10,9(4,2)$ \\
IMC (kg/m²) & $25,2(4,6)$ & $26,0(4,2)$ & $24,7(4,8)$ \\
Número de refeições & $4,0(0,9)$ & $4,0(1,1)$ & $4,0(0,9)$ \\
\hline
\end{tabular}

Tabela 2

Frequência (\%) de indicadores de consumo de alimentos e de bebidas, estimados com base em duas entrevistas telefônicas (VIGITEL e PR), realizadas com os mesmos indivíduos, segundo sexo. Belém, Pará, Brasil, 2009

\begin{tabular}{|c|c|c|c|}
\hline Indicadores/Sexo & VIGITEL & PR & Kappa \\
\hline \multicolumn{4}{|c|}{ Consumo de frutas, legumes e verduras $\geq 5$ dias da semana } \\
\hline Masculino & 22,2 & 38,9 & 0,366 \\
\hline Feminino & 28,1 & 57,8 & 0,093 \\
\hline Total & 26,0 & 51,0 & 0,188 \\
\hline \multicolumn{4}{|c|}{ Consumo habitual de carne vermelha com gordura visível ou frango com pele } \\
\hline Masculino & 47,2 & 47,2 & 0,003 \\
\hline Feminino & 15,6 & 34,4 & 0,284 \\
\hline Total & 27,0 & 39,0 & 0,199 \\
\hline \multicolumn{4}{|c|}{ Consumo habitual de leite com teor integral de gordura } \\
\hline Masculino & 66,7 & 72,2 & 0,087 \\
\hline Feminino & 67,2 & 82,2 & 0,354 \\
\hline Total & 67,0 & 79,0 & 0,253 \\
\hline \multicolumn{4}{|c|}{$\begin{array}{l}\text { Consumo de um ou mais copos de refrigerante com açúcar em } \geq 5 \text { dias da } \\
\text { semana }\end{array}$} \\
\hline Masculino & 13,9 & 5,6 & 0,224 \\
\hline Feminino & 18,8 & 15,6 & 0,342 \\
\hline Total & 17,0 & 12,0 & 0,318 \\
\hline \multicolumn{4}{|c|}{ Consumo de mais de cinco (homem) ou mais de quatro (mulher) doses de } \\
\hline \multicolumn{4}{|c|}{ bebidas alcoólicas em uma mesma ocasião nos últimos 30 dias } \\
\hline Masculino & 41,7 & 25,0 & 0,152 \\
\hline Feminino & 7,8 & 1,6 & 0,027 \\
\hline Total & 20,0 & 10,0 & 0,231 \\
\hline
\end{tabular}

PR: padrão de referência (três recordatórios de 24 horas em dias diferentes); VIGITEL: Vigilância de Fatores de Risco e Proteção para Doenças Crônicas por Inquérito Telefônico.

no VIGITEL e nos três R24h-PR, realizados com os mesmos indivíduos. A frequência do indicador de proteção para DCNT (consumo de frutas, legumes e verduras) foi maior quando estimado pelo PR, do que quando pelo VIGITEL ( $51 \%$ vs. $26 \%$ ). Para os indicadores de risco para DCNT, a frequência do consumo habitual de gordura sa- turada foi maior quando estimado pelo PR, do que pelo VIGITEL (39\% vs. $27 \%$ e 79 vs. $67 \%$, respectivamente para consumo de carne vermelha com gordura visível ou frango com pele e leite com teor integral de gordura). Já o consumo de refrigerante foi menor quando estimado com base no PR, do que no VIGITEL ( $12 \%$ vs. $17 \%$ ); 
o mesmo ocorre com o consumo abusivo de bebidas alcoólicas (10\% vs. 20\%). Quanto aos níveis de concordância entre o VIGITEL e o PR, o coeficiente kappa indicou concordância fraca (abaixo de 0,21 ) para os indicadores de proteção (consumo de frutas, legumes e verduras) e de risco (consumo de gordura visível nas carnes) e concordância razoável $(0,21$ a 0,40$)$ para os demais indicadores de risco para DCNT.

A sensibilidade das entrevistas realizadas pelo VIGITEL, a partir da classificação dos indivíduos feita pelo PR, variou entre 35,3 (consumo de frutas, legumes e verduras) e $73,4 \%$ (consumo habitual de leite com teor integral de gordura). A especificidade do VIGITEL, a partir da mesma classificação, variou entre 57,1 (consumo habitual de leite com teor integral de gordura) e 87,5\% (consumo de refrigerante com açúcar). Observase, ainda, alta porcentagem de falsos positivos, isto é, indivíduos sem consumo na entrevista de validação, mas que se classificaram como consumidores no VIGITEL, no que se refere aos refrigerantes $(64,7 \%)$ e ao consumo abusivo de bebidas alcoólicas (75\%), como também ao consumo de frutas, legumes e verduras e ao hábito de consumo de carnes com gorduras, que apresentaram porcentagens acima de $30 \%$. Inversamente, o consumo habitual de leite com teor integral de gordura apresentou alta proporção de falsos negativos $(63,6 \%)$, isto é, de indivíduos com consumo na entrevista de validação, mas que se classificaram como não consumidores no VIGITEL; para consumo de frutas, legumes e verduras e hábito de consumo de carnes com gorduras, encontraram-se valores acima de $30 \%$. Notam-se, ainda, diferenças substanciais entre os sexos: para consumo de frutas, legumes e verduras e consumo abusivo de bebidas alcoólicas, a concordância é maior entre os homens; para os demais indicadores, a concordância é maior entre as mulheres (Tabela 3 ).

A Tabela 4 apresenta comparação entre a mediana da frequência semanal de consumo de alimentos e de bebidas associados à proteção ou

Valores de sensibilidade, especificidade, falsos positivos e falsos negativos dos indicadores de consumo de alimentos e de bebidas, estimados com base em duas entrevistas telefônicas (VIGITEL e PR), realizadas com os mesmos indivíduos, segundo sexo. Belém, Pará, Brasil, 2009.

\begin{tabular}{|c|c|c|c|c|}
\hline Indicadores/Sexo & $\mathbf{S}$ & $\mathbf{E}$ & $\mathrm{F}+$ & F- \\
\hline \multicolumn{5}{|c|}{ Consumo de frutas, legumes e verduras $\geq 5$ dias da semana } \\
\hline Masculino & 42,9 & 90,9 & 25,0 & 28,6 \\
\hline Feminino & 32,4 & 77,8 & 33,3 & 54,3 \\
\hline Total & 35,3 & 83,7 & 30,8 & 44,6 \\
\hline \multicolumn{5}{|c|}{ Consumo habitual de carne vermelha com gordura visível ou frango com pele } \\
\hline Masculino & 47,1 & 52,6 & 52,9 & 47,4 \\
\hline Feminino & 31,8 & 92,9 & 30,0 & 27,8 \\
\hline Total & 38,5 & 80,3 & 44,4 & 32,9 \\
\hline \multicolumn{5}{|c|}{ Consumo habitual de leite com teor integral de gordura } \\
\hline Masculino & 69,2 & 40,0 & 25,0 & 66,7 \\
\hline Feminino & 75,5 & 72,7 & 7,0 & 61,9 \\
\hline Total & 73,4 & 57,1 & 13,4 & 63,6 \\
\hline \multicolumn{5}{|c|}{ Consumo de um ou mais copos de refrigerante com açúcar em $\geq 5$ dias da semana } \\
\hline Masculino & 50,0 & 88,2 & 80,0 & 3,2 \\
\hline Feminino & 50,0 & 87,0 & 58,3 & 9,6 \\
\hline Total & 50,0 & 87,5 & 64,7 & 7,2 \\
\hline \multicolumn{5}{|c|}{ Consumo de mais de 5 (homem) ou mais de 4 (mulher) doses de bebidas } \\
\hline \multicolumn{5}{|c|}{ alcoólicas em uma mesma ocasião nos últimos 30 dias } \\
\hline Masculino & 55,6 & 63,0 & 66,7 & 19,0 \\
\hline Feminino & 0,0 & 92,1 & 100,0 & 1,7 \\
\hline Total & 50,0 & 83,3 & 75,0 & 6,3 \\
\hline
\end{tabular}

E: especificidade; F-: falso negativo; F+: falso positivo; PR: padrão de referência (três recordatórios de 24 horas em dias diferentes); S: sensibilidade; VIGITEL: Vigilância de Fatores de Risco e Proteção para Doenças Crônicas por Inquérito Telefônico. 
Tabela 4

Frequência semanal de consumo estimado de alimentos e bebidas com base em três recordatórios de 24 horas, realizados por inquérito telefônico, classificados pelo VIGITEL como expostos ou não expostos à frequência crítica de consumo *. Belém, Pará, Brasil, 2009.

\begin{tabular}{|c|c|c|c|}
\hline \multicolumn{4}{|c|}{ Mediana da frequência semanal de consumo (dias por semana) } \\
\hline Indicadores/Sexo & Expostos & Não expostos & Valor de $p$ \\
\hline \multicolumn{4}{|c|}{ Frutas, legumes e verduras } \\
\hline Masculino & 2,5 & 1,3 & 0,036 \\
\hline Feminino & 2,8 & 1,9 & 0,041 \\
\hline Total & 2,7 & 1,7 & 0,004 \\
\hline \multicolumn{4}{|c|}{ Carne ou frango com excesso de gordura } \\
\hline Masculino & 2,7 & 1,8 & 0,363 \\
\hline Feminino & 2,8 & 1,0 & 0,013 \\
\hline Total & 2,7 & 1,2 & 0,002 \\
\hline \multicolumn{4}{|c|}{ Leite com teor integral de gordura } \\
\hline Masculino & 4,8 & 3,3 & 0,062 \\
\hline Feminino & 6,1 & 3,0 & $<0,001$ \\
\hline Total & 5,6 & 3,1 & $<0,001$ \\
\hline \multicolumn{4}{|c|}{ Refrigerante com açúcar } \\
\hline Masculino & 1,9 & 1,7 & 0,929 \\
\hline Feminino & 3,8 & 1,9 & 0,010 \\
\hline Total & 3,2 & 1,9 & 0,029 \\
\hline \multicolumn{4}{|l|}{ Bebidas alcoólicas } \\
\hline Masculino & 0,9 & 0,4 & 0,485 \\
\hline Feminino & 0,0 & 0,1 & 0,961 \\
\hline Total & 0,7 & 0,2 & 0,014 \\
\hline
\end{tabular}

* Frequência crítica de consumo corresponde a cinco ou mais dias da semana para frutas, legumes e verduras e para refrigerante com açúcar; pelo menos dois dias por semana de excesso de gordura (carne ou frango) e de leite integral; e pelo menos um dia por semana de consumo de bebidas alcoólicas.

VIGITEL: Vigilância de Fatores de Risco e Proteção para Doenças Crônicas por Inquérito Telefônico.

risco de DCNT, estimado a partir dos três R24h, com a frequência de indivíduos classificados pelo VIGITEL como expostos ou não expostos aos indicadores. Verifica-se que a mediana da frequência semanal de consumo (dias por semana) foi maior em ambos os sexos apenas para consumo de frutas, legumes e verduras no grupo classificado pelo VIGITEL como expostos à frequência crítica de consumo. Para os demais indicadores, à exceção do consumo abusivo de bebidas alcoólicas, apenas as mulheres apresentaram maior consumo no grupo classificado pelo VIGITEL como expostos à frequência crítica de consumo, com reflexo direto no consumo combinado dos dois sexos. Para o consumo abusivo de bebidas alcoólicas, apenas os dois sexos combinados mostrou maior consumo no grupo classificado pelo VIGITEL como expostos à frequência crítica.

\section{Discussão}

Um dos grandes desafios do uso de métodos simplificados para avaliar o consumo alimentar, como o utilizado no VIGITEL, é o fornecimento de dados compatíveis com métodos considerados de referência. Comumente, a validação de inquéritos dietéticos se dá pela sua correlação com parâmetros bioquímicos e/ou pela sua correlação com outro instrumento de consumo alimentar mais exato, considerado padrão de referência $15,16,17,18$.

Pela dificuldade de se encontrar um padrãoouro consensual para a validação dos indicadores estudados pelo VIGITEL, realizou-se a validação relativa, mediante o uso do R24h, método bastante usado por vários autores, como padrão de referência 7,8,19,20. O R24h em diferentes dias da semana tem sido utilizado pela fácil aplicabilidade e pelo fornecimento de informações detalhadas sobre os tipos de alimentos e bebidas consu- 
midos, bem como suas quantidades, o que torna útil seu emprego em inquéritos populacionais e, também, em estudos de validação de métodos mais simplificados, como o do VIGITEL 21,22,23.

A realização de pelo menos três $\mathrm{R} 24 \mathrm{~h}$ em diferentes dias da semana é o procedimento mais usual como método de referência para inquéritos de frequência do consumo de alimentos 24,25. Todavia, a aplicação de apenas três R24h para validação do consumo habitual pode ter sido uma limitação no nosso estudo, como também foi observado por Zanolla et al. ${ }^{26}$ em estudo de validação de questionário de frequência alimentar com adultos. O ideal seria a ampliação do período de aplicação dos recordatórios para uma semana, ou ainda para um maior período (no caso específico do indicador consumo abusivo de bebidas alcoólicas, o período de referência é de 30 dias), na tentativa de reduzir o eventual erro decorrente da variância intrapessoal 16,27. Por outro lado, a repetição de inquéritos pode alterar o consumo ou as respostas, como "efeito de treinamento"; no entanto, a variação intra-indivíduos (dados não mostrados) nestes três recordatórios não sofreu este efeito. Dos itens avaliados, apenas o consumo de leite integral sofreu redução gradativa e significante nos dois sexos, mas isso se deu em virtude da redução do consumo de leite como um todo, e não por causa da substituição do leite integral por outro de menor teor de gorduras saturadas.

Neste estudo, a validade relativa dos indicadores foi analisada coletiva e individualmente, utilizando três R24h como método de referência.

No padrão de referência, a avaliação coletiva verificou a concordância entre os dois inquéritos e evidenciou a subestimação na frequência de todos os indicadores do consumo de alimentos e bebidas, à exceção do consumo regular de refrigerante com açúcar e do consumo abusivo de bebidas alcoólicas. Adicionalmente, apenas para os dois sexos combinados, a comparação da mediana da frequência semanal de consumo foi maior e estatisticamente significativa no grupo classificado pelo VIGITEL como expostos à frequência crítica de consumo. Individualmente, considerando conjuntamente os dois sexos, foram encontrados valores baixos ou regulares (entre $35,3 \%$ e $73,4 \%$ ) para sensibilidade, e valores regulares ou satisfatórios (entre 57,1\% e $87,5 \%)$ para especificidade. Valores elevados de falsos positivos foram encontrados para consumo de refrigerante com açúcar e bebidas alcoólicas: $64,7 \%$ dos indivíduos estudados responderam no VIGITEL consumir refrigerante com açúcar, enquanto nos R24h este produto não apareceu; no caso de bebidas alcoólicas, $75 \%$ responderam positivamente no VIGITEL, mas o produto não apareceu nos recordatórios. Quanto ao consumo de leite com teor integral de gordura, o número de falsos negativos foi de $63,6 \%$, representando os indivíduos que consumiram este alimento conforme identificado nos três recordatórios, mas que se disseram não consumidores no VIGITEL.

O teste kappa 11,12 é utilizado para comparar as categorias de frequência de consumo de alimentos e bebidas, medida por dois métodos, quando houver variáveis dicotômicas ${ }^{28}$. O grau de concordância individual entre as duas entrevistas (VIGITEL e PR) evidenciou concordância de fraca a razoável para os indicadores de consumo de alimentos e bebidas. Contudo, as altas porcentagens de concordância coletiva configuram um paradoxo que pode ter ocorrido pela inadequação da estimativa kappa 29.

A subestimação do consumo na entrevista do VIGITEL pode ser explicada por menor valorização dos alimentos estudados na composição da dieta regional, por exemplo: legumes e verduras entram na composição do prato principal, cozidos junto com o feijão, com o arroz, com a carne e/ou na forma de sopa. Situações que são mais bem identificadas pelo R24h. O mesmo acontece com o consumo de gordura em excesso nas carnes, principalmente o charque, que é cozido junto com o feijão e/ou sopa, e não identificado no VIGITEL como alimento por si só. Já o consumo de leite integral pode ter sido maior pela simples oportunidade de aparecimento nos três recordatórios e viés de memória no VIGITEL, uma vez que a recomendação para o intervalo entre as entrevistas de dois métodos não deve ultrapassar 15 dias 8, e no estudo de validação do VIGITEL o intervalo chegou até 30 dias.

O uso do R24h como método de referência pode identificar a subestimação dos itens avaliados, como se verificou no estudo de São Paulo 5. Esse método se limita a alguns dias e permite maior aprofundamento dos alimentos consumidos, isoladamente ou como parte dos ingredientes do prato principal, enquanto o VIGITEL questiona sobre o consumo usual.

A existência de poucas investigações sobre validação de consumo alimentar no Brasil dificulta a comparação dos resultados obtidos. Assim, recortando os estudos existentes para os mesmos alimentos e bebidas avaliados na presente pesquisa, observamos inconsistência na validação do consumo de frutas, legumes e verduras, como apresentado a seguir.

Estudos desenvolvidos com escolares, no Balneário de Camburiú (Santa Catarina) ${ }^{30}$, mostraram concordância substancial para frutas e para legumes e verduras, em 2005, e concordância substancial para frutas e moderada para le- 
gumes e verduras, em 2007, o que resultou em baixa sensibilidade (36\%) para legumes e verduras 14. O mesmo foi identificado no presente estudo com adultos. Salienta-se que os estudos de Camburiú tiveram a observação do consumo como método de referência e o registro em cartilha como teste.

Voci et al. 25, em estudo de validação de questionário de frequência do consumo de alimentos, usaram o R24h como PR, em Piracicaba (São Paulo), no ano de 2004, identificando, em adolescentes de 11 a 15 anos de idade, superestimação do consumo de frutas, legumes e verduras e de bebidas alcoólicas, por um lado, e subestimação de refrigerante com açúcar, por outro, cujas concordâncias foram, respectivamente, razoável, moderada, fraca e razoável. Estes achados foram contrários aos observados no presente estudo, exceto quanto ao consumo de bebidas alcoólicas.

A investigação de São Paulo 5 avaliou, em 2005, a validação de método semelhante ao empregado neste estudo na população adulta. Os autores apontaram subestimação na frequência do consumo de carnes com gordura e leite integral e superestimação na frequência do consumo de frutas, legumes e verduras, de refrigerante com açúcar e bebidas alcoólicas. À exceção de frutas, legumes e verduras, identificamos a mesma situação em Belém. Mais uma vez, justificamos a subestimação de frutas, legumes e verduras na entrevista do VIGITEL pela simplicidade e não aprofundamento das questões sobre estes alimentos de forma associada ao hábito alimentar regional, o que foi possível com o R24h. Do mesmo modo, as medianas do consumo dos itens estudados foram maiores no grupo identificado como exposto.

Em estudos de validação, a comparação de resultados é limitada pelas diferenças no tipo de questionário utilizado para avaliar o consumo alimentar, as características das amostras, a forma de coleta dos dados e as análises estatís- ticas 14,30 . No presente estudo, a amostra estudada foi a mesma, assim como a técnica de coleta de dados - por entrevista telefônica -, e os testes estatísticos utilizados foram os recomendados pela literatura, porém o instrumento foi diferente. Na entrevista do VIGITEL as perguntas eram diretas, com uma única alternativa de resposta (exemplo: "Em quantos dias da semana, o (a) senhor (a) costuma tomar leite? A) 1 a 2, B) 3 a 4, C) $5 a$ 6, D) todos, E) quase nunca, F) nunca"; se a resposta fosse qualquer alternativa, entre A a D, perguntava-se: "Quando o (a) senhor (a) toma leite, que tipo de leite costuma tomar? A) integral, B) desnatado ou semi-desnatado, C) os dois tipos, D) não sabe"); no R24h, por sua vez, iniciava-se com um relato espontâneo dos alimentos e bebidas consumidos nas últimas 24 horas e recorria-se à evocação da memória, quando algum dos itens avaliados não fosse citado.

Diante das limitações observadas em ambos os métodos (VIGITEL e R24h), não se pode concluir, com os resultados apresentados, que o questionário de consumo alimentar aplicado peloVIGITEL não seja um bom indicador do padrão alimentar da população de Belém, uma vez que o padrão de referência também apresenta limitações. Diante disso, como as médias das frequências de consumo são, em geral, maiores no grupo classificado como exposto à frequência crítica de consumo no VIGITEL, justifica-se o uso deste como instrumento de monitoramento dos fatores dietéticos de risco e proteção para doenças crônicas não transmissíveis. Alterações no formato das questões do VIGITEL têm sido observadas ao longo dos seus quatro anos de história 10, principalmente quanto ao consumo de frutas, legumes e verduras, devido à sua fragilidade em distinguir a população exposta e não exposta ao consumo crítico desses alimentos. Estudos de validação dos indicadores de consumo de alimentos e bebidas são ainda necessários para melhor compreensão desses marcadores nas diferentes regiões geográficas do país. 


\section{Resumo}

O objetivo deste trabalho foi avaliar a validade relativa dos indicadores de consumo de alimentos e bebidas obtidos pelo sistema de vigilância por entrevista telefônica (VIGITEL). Foi avaliada uma amostra aleatória $(n=100)$ da amostra total de aproximadamente dois mil adultos, estudada pelo sistema, em 2009, em Belém, Pará, Brasil. Os indicadores avaliados foram fatores de proteção (consumo adequado de frutas, legumes e verduras) e de risco (consumo de gordura saturada, refrigerante e bebidas alcoólicas) para doenças crônicas não transmissiveis. Compararam-se os resultados obtidos a partir de entrevista telefônica e de três recordatórios de 24 horas (padrão de referência). O padrão de referência evidenciou subestimação na frequência dos indicadores, à exceção de refrigerantes e bebidas alcoólicas. As médias das frequências do consumo foram, em geral, maiores no grupo exposto (entrevistados pelo VIGITEL). Não se pode concluir que o VIGITEL não seja um bom indicador de consumo, pois o padrão de referência também apresenta limitações, portanto o uso daquele como instrumento de monitoramento é justificável.

Consumo de Alimentos; Inquérito sobre Dietas; Vigilância Epidemiológica

\section{Referências}

1. World Health Organization. Diet, nutrition and prevention of chronic diseases: report of a joint WHO/FAO expert consultation. Geneva: World Health Organization; 2003.

2. World Health Organization. The world health report 2002: reducing risks, promoting healthy life. Geneva: World Health Organization; 2002.

3. Departamento de Análise de Situação em Saúde, Secretaria de Vigilância em Saúde, Ministério da Saúde. Saúde Brasil 2007: uma análise da situação da saúde. Brasília: Ministério da Saúde; 2008.

4. Monteiro CA, Moura EC, Jaime PC, Lucca A, Florindo AA, Figueiredo ICR, et al. Monitoramento de fatores de risco para as doenças crônicas por entrevistas telefônicas. Rev Saúde Pública 2005 39:47-57.

5. Monteiro CA, Moura EC, Jaime PC, Claro RM. Validade de indicadores do consumo de alimentos e bebidas obtidos por inquérito telefônico. Rev Saúde Pública 2008; 42:582-9.

\section{Colaboradores}

A. C. M. Neves e L. A. A. Gonzaga realizaram a tabulação e organização dos dados, revisão bibliográfica e a redação do manuscrito. I. B. G. Martens contribuiu com a definição do desenho e revisão do manuscrito. E. C. Moura participou da definição do desenho metodológico, análise estatística e revisão do manuscrito.

\section{Agradecimentos}

Aos colegas Aline de Paula, Aline de Souza, Daiana Almeida, Francília Silva, Josiana Moreira, Liliane Salla, Marcela Souza, Sheila Martins, Tayana de Miranda e Victor Vieira, pelo auxílio na coleta de dados. Às nutricionistas Cláudia Dutra, Naiza de Sá e Sara Silva, pelo apoio logístico.
6. Willett WC. Future directions in the development of food-frequency questionnaires. Am J Clin Nutr 1994; 59:171-4.

7. Lopes ACS, Caiaffa WT, Mingoti SA, Lima-Costa MFF. Ingestão alimentar em estudos epidemiológicos. Rev Bras Epidemiol 2003; 6:209-19.

8. Willett W, Lenart E. Reproducibility and validity of food-frequency questionnaires. In: Willett W, editor. Nutritional epidemiology. 2nd Ed. New York: Oxford University Press; 1998. p. 101-47. (Monographs in Epidemiology and Biostatistics, 30).

9. Moura EC, Malta DC, Morais Neto OL, Monteiro CA. Prevalence and social distribution of risk factors for chronic noncommunicable diseases in Brazil. Rev Panam Salud Pública 2009; 26:17-22.

10. Secretaria de Vigilância em Saúde, Ministério da Saúde. VIGITEL Brasil 2008. Vigilância de fatores de risco e proteção para doenças crônicas por inquérito telefônico. Brasília: Ministério da Saúde; 2009. 
11. Kelsey JL, Whittemore AS, Evans AS, Thompson WD. Methods in observational epidemiology. 2nd Ed. New York: Oxford University Press; 1996.

12. Masson LF, McNeill G, Tomany JO, Simpson JA, Peace HS, Wei L, et al. Statistical approaches for assessing the relative validity of a food-frequency questionnaire: use of correlation coefficients and the kappa statistic. Public Health Nutr 2003; 6 : 313-21.

13. Szklo M, Nieto FJ. Epidemiology: beyond the basics. Am J Epidemiol 2001; 153:596-603.

14. Assis MAA, Benedet J, Kerpel R, Vasconcelos FAG, Di Pietro PF, Kupek E. Validação da terceira versão do Questionário Alimentar do Dia Anterior (QUADA-3) para escolares de 6 a 11 anos. Cad Saúde Pública 2009; 25:1816-26.

15. Jackson M, Walker S, Cade J, Forrester T, Cruickshank JK, Wilks R. Reproducibility and validity of a quantitative food-frequency questionnaire among Jamaicans of African origin. Public Health Nutr 2001; 4:971-80.

16. Slater B, Philippi ST, Marchioni DML, Fisberg RM. Validação de questionários de frequência alimentar - QFA: considerações metodológicas. Rev Bras Epidemiol 2003; 3:200-8.

17. Costa AGV, Priore SE, Sabarense CM, Franceschini SCC. Questionário de frequência de consumo alimentar e recordatório de 24 horas: aspectos metodológicos para avaliação de ingestão de lipídeos. Rev Nutr 2006; 19:631-41.

18. Barbosa KB, Lima-Rosado LE, Franceschini SC, Priore SE. Instrumentos de inquérito dietético utilizados na avaliação do consumo alimentar em adolescentes: comparação entre métodos. Arch Latinoam Nutr 2007; 57:43-50.

19. Salvo VLMA, Gimeno SGA. Reprodutibilidade e validade do questionário de frequência de consumo de alimentos. Rev Saúde Pública 2002; 36:505-12.

20. Kaaks R, Riboli E. Validation and calibration of dietary intake measurements in the EPIC Project: methodological considerations. Int J Epidemiol 1997; 26:S15-25.

21. Cardoso MA, Stocco PR. Desenvolvimento de um questionário quantitativo de frequência alimentar em imigrantes japoneses e seus descendentes residentes em São Paulo, Brasil. Cad Saúde Pública 2000; 16:107-14.
22. Cavalcante AAM, Priore SE, Franceschini SCC. Estudos de consumo alimentar: aspectos metodológicos gerais e o seu emprego na avaliação de crianças e adolescentes. Rev Bras Saúde Matern Infant 2004; 4:229-40.

23. Morimoto JM, Latorre MRDO, César CLG, Carandina L, Barros MBA, Goldbaum M, et al. Fatores associados à qualidade da dieta de adultos residentes na região metropolitana de São Paulo, Brasil, 2002. Cad Saúde Pública 2008; 24:169-78.

24. Millen AE, Midthune D, Thompson FE, Kipnis V, Subar AF. The national cancer institute diet history questionnaire: validation of pyramid food servings. Am J Epidemiol 2005; 163:279-88.

25. Voci SM, Enes CC, Slater B. Validação do questionário de frequência alimentar para adolescentes (QFAA) por grupos de alimentos em uma população de escolares. Rev Bras Epidemiol 2008; 11: 561-72.

26. Zanolla AF, Olinto MTA, Henn RL, Wahrlich V, Anjos LA. Avaliação de reprodutibilidade e validade de um questionário de frequência alimentar em adultos residentes em Porto Alegre, Rio Grande do Sul, Brasil. Cad Saúde Pública 2009; 25:840-8.

27. Sales RL, Silva MMS, Costa NMB, Euclydes MP, Eckhardt VF, Rodrigues CMA, et al. Desenvolvimento de um inquérito para avaliação da ingestão alimentar de grupos populacionais. Rev Nutr 2006; 19:539-52.

28. Cade J, Thompson R, Burley V, Warm D. Development, validation and utilization of food-frequency questionnaires: a review. Public Health Nutr 2002; 5:567-87.

29. Feinstein AR, Cicchetti DV. High agreement but low kappa: I. The problems of two paradoxes. J Clin Epidemiol 1990; 43:543-9.

30. Assis MAA, Guimarães D, Calvo MCM, Barros MVG, Kupek E. Reprodutibilidade e validade de questionário de consumo alimentar para escolares. Rev Saúde Pública 2007; 41:1054-7.

Recebido em 13/Nov/2009

Versão final reapresentada em 16/Jun/2010

Aprovado em 21/Jun/2010 